\title{
Manipulating the position of DNA expression cassettes using location tags fused to dCas9 (Cas9-Lag) to improve metabolic pathway efficiency
}

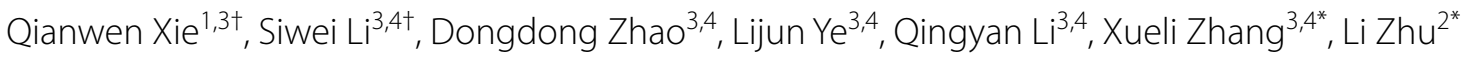
and Changhao $\mathrm{Bi}^{3,4^{*}}$ (D)

\begin{abstract}
Background: Deactivated Cas9 (dCas9) led to significant improvement of CRISPR/Cas9-based techniques because it can be fused with a variety of functional groups to form diverse molecular devices, which can manipulate or modify target DNA cassettes. One important metabolic engineering strategy is to localize the enzymes in proximity of their substrates for improved catalytic efficiency. In this work, we developed a novel molecular device to manipulate the cellular location of specific DNA cassettes either on plasmids or on the chromosome, by fusing location tags to dCas9 (Cas9-Lag), and applied the technique for synthetic biology applications. Carotenoids like $\beta$-carotene serve as common intermediates for the synthesis of derivative compounds, which are hydrophobic and usually accumulate in the membrane compartment.

Results: Carotenoids like $\beta$-carotene serve as common intermediates for the synthesis of derivative compounds, which are hydrophobic and usually accumulate in the membrane components. To improve the functional expression of membrane-bound enzymes and localize them in proximity to the substrates, Cas9-Lag was used to pull plasmids or chromosomal DNA expressing carotenoid enzymes onto the cell membrane. For this purpose, dCas9 was fused to the E. coli membrane docking tag GlpF, and gRNA was designed to direct this fusion protein to the DNA expression cassettes. With Cas9-Lag, the zeaxanthin and astaxanthin titer increased by $29.0 \%$ and $26.7 \%$ respectively. Due to experimental limitations, the electron microscopy images of cells expressing Cas9-Lag vaguely indicated that GlpFCas9 might have pulled the target DNA cassettes in close proximity to membrane. Similarly, protein mass spectrometry analysis of membrane proteins suggested an increased expression of carotenoid-converting enzymes in the membrane components.
\end{abstract}

Conclusion: This work therefore provides a novel molecular device, Cas9-Lag, which was proved to increase zeaxanthin and astaxanthin production and might be used to manipulate DNA cassette location.

Keywords: dCas9, Complex localization, Astaxanthin, Carotenoids, Escherichia coli

*Correspondence: zhang_xl@tib.cas.cn; jewly54@126.com; bi_ch@tib.cas.cn

${ }^{\dagger}$ Qianwen Xie and Siwei Li have contributed equally to this work

${ }^{2}$ State Key Laboratory of Pathogen and Biosecurity, Beijing Institute of Biotechnology, Beijing 100071, China

${ }^{3}$ Tianjin Institute of Industrial Biotechnology, Chinese Academy of Sciences, Tianjin 300308, P. R. China

Full list of author information is available at the end of the article

\section{Introduction}

The RNA-guided Cas9 nuclease has been developed into a powerful genome editing tool in recent years [1-3]. The Clustered Regularly Interspersed Short Palindromic Repeats CRISPR/Cas9 system is able to recognize and

c) The Author(s) 2020. This article is licensed under a Creative Commons Attribution 4.0 International License, which permits use, sharing, adaptation, distribution and reproduction in any medium or format, as long as you give appropriate credit to the original author(s) and the source, provide a link to the Creative Commons licence, and indicate if changes were made. The images or other third party material in this article are included in the article's Creative Commons licence, unless indicated otherwise in a credit line to the material. If material is not included in the article's Creative Commons licence and your intended use is not permitted by statutory regulation or exceeds the permitted use, you will need to obtain permission directly from the copyright holder. To view a copy of this licence, visit http://creativecommons.org/licenses/by/4.0/. The Creative Commons Public Domain Dedication waiver (http://creativecommons.org/publicdomain/zero/1.0/) applies to the data made available in this article, unless otherwise stated in a credit line to the data. 
make double-strand breaks at target sequences based solely on a guide RNA $[4,5]$. More recently, the invention of deactivated Cas9 further expanded the scope of this technique, allowing the protein to specifically bind to designed DNA sequences without inducing strand breaks. A variety of functional components were fused to dCas9 to form molecular devices, which implemented novel biological functions aimed at the target DNA, such as transcriptional blockage, epigenetic modification and gene expression regulation [4-6]. For example, the $\omega$ group of RNA polymerase was fused to dCas9 to form a complex which was guided to promoter regions of $E$. coli genes to activate transcription initiation [6,7]. Recently, a new family of CRISPR-based genome editing methods, named base editor techniques, were developed by fusing the PmCDA1 activation-induced cytidine deaminase (AID) from sea lamprey to dCas9 to form a molecular device that can perform targeted base editing [8]. In this work, we developed a novel molecular device to manipulate the cellular position of specific DNA cassettes either on plasmids or on the chromosome, by fusing location tags to dCas9 (Cas9-Lag) and applied the technique for synthetic biology tasks (Fig. 1a).

Due to the medical and preventive value of carotenoids, metabolic engineering for the heterologous production of carotenoids has been a research hotspot for decades, and numerous novel techniques have led to advances in this field $[9,10]$. In the heterologous carotenoid synthesis pathway (Fig. 1b), IPP and DMAPP are synthesized by the MVA or MEP pathway, and then sequentially converted to $\beta$-carotene by farnesyl diphosphate synthase (IspA), geranylgeranyl diphosphate synthase (CrtE), phytoene synthase $(\mathrm{CrtB})$, phytoene desaturase (CrtI), and lycopene $\beta$-cyclase $(\mathrm{CrtY})[11,12]$. Astaxanthin is derived from $\beta$-carotene via
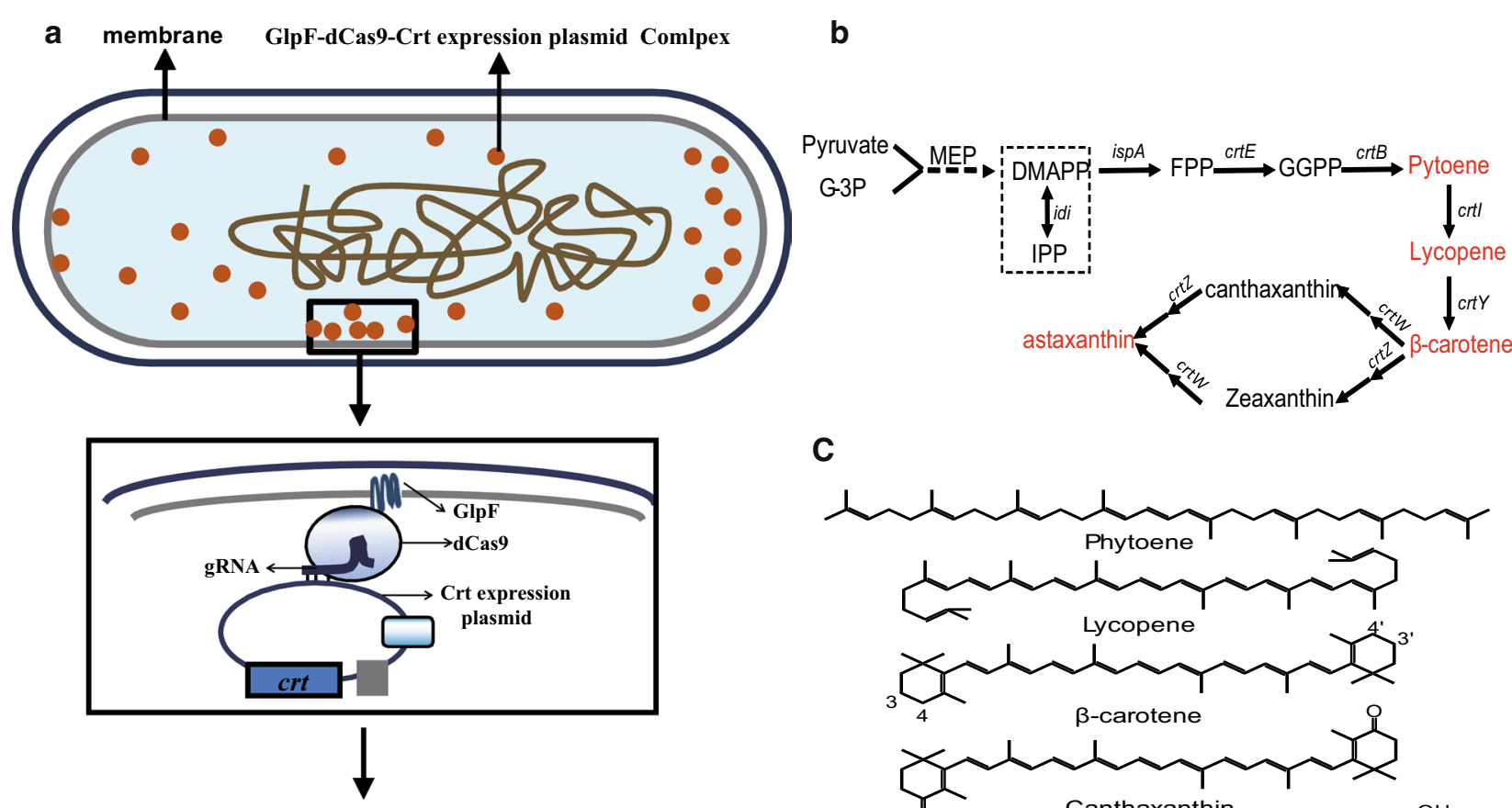

C
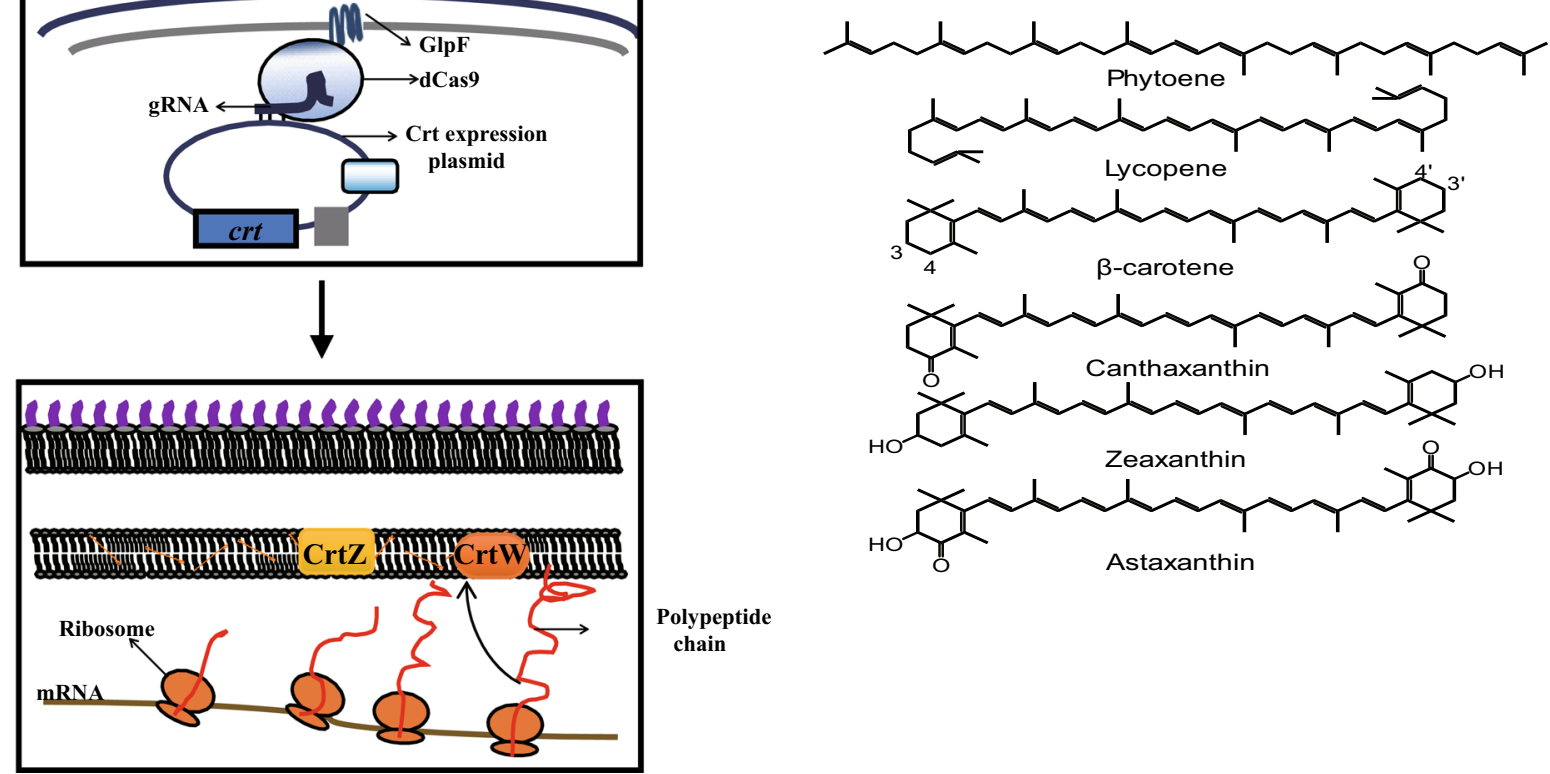

Fig. 1 a Manipulate position of expression DNA cassettes strategies for improving Carotenoids production. $\mathbf{b}$ Schematic diagram of the heterologous synthesis pathway for the production of carotenoids. c The Molecular Formula diagram of the various carotenoids 
reactions catalyzed by the two enzymes CrtZ (3,3'-hydroxylase) and CrtW (4,4'-oxygenase) [13, 14].

Most of the intermediates upstream of the synthetic pathway are water-soluble molecules, while the downstream compounds are mostly hydrophobic (Fig. 1c) and were proved to accumulate near the membrane $[15,16]$. How to efficiently organize the catalytic reactions of each step and increase the catalytic efficiency of the enzymes in the synthetic pathway is an important issue in the construction of carotenoids cell factories. Consequently, the spatial optimization of isoprenoid synthesis pathways has attracted the attention of many researchers. For instance, by targeting sesquiterpene synthases, such as valencene or amorphadiene synthase, and heterologous FDP synthase to the mitochondria, the yield of valencene and amorphadiene in Saccharomyces cerevisiae was increased 8- and 20-fold [17]. The entire MVA pathway was localized into the mitochondria to improve the utilization of acetyl-CoA for isoprenoid production, by dual metabolic engineering of cytoplasmic and mitochondrial, the production of isoprene in S. cerevisiae was respectively increased 2.1- and 1.6-fold relative to the recombinant strains with solely mitochondrial or cytoplasmic engineering [18].

In our own earlier study, we investigated the cell membrane localization of single or multiple enzymes, as well as entire metabolic pathways of carotenoid synthesis, and discussed the relationship between catalytic efficiencies and different localization strategies [19]. The focus of these strategies was to bring the enzymes to their favorable environment and in close to their substrates. However, signal peptides or membrane proteins induced localization may sometimes too complex to implement for multiple enzymes, and the formation of fusion proteins may affect the conformation and function of these proteins.

In this work, to localize plasmids or chromosomal cassettes expressing carotenoid enzymes to the cell membrane compartment, dCas9 was fused with the $E$. coli membrane docking tag GlpF [20], and gRNA was designed to direct this fusion protein to the targeted DNA cassette. By the pull of dCas9-Lag, plasmids of $c r t Z$ and $c r t W$ might be in close proximity to membrane, which bring them close to their substrates, and the functional expression of the membrane binding enzymes were increased (Fig. 1a). Finally, the impact of the localization on carotenoids production was discussed.

\section{Results}

\section{The GlpF-dCas9/gRNA complex specifically binds to the target plasmid}

To construct a molecular device to manipulate the cellular localization of a specific DNA cassette, GlpF was fused to the amino terminus of dCas9 with a short flexible linker (GGGS). Since dCas9 was proved to decrease

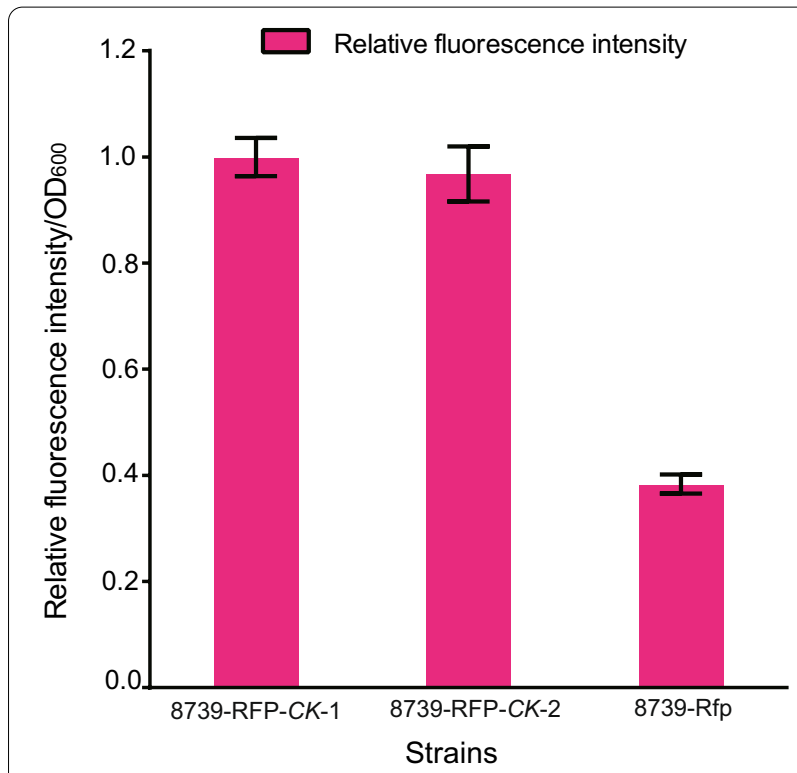

Fig. 2 GlpF-dCas9/gRNA specifically binds to target plasmid. Expression levels of pBAD-RFP under different gene manipulation was illustrated. Compared with 8739-RFP-CK1 and 8739-RFP-CK2, the fluorescence intensity of RFP decreased significantly for the binding of GlpF-dCas9/gRNA complex to the $r f p$ N terminal region. 8739-RFP(8739 co-transformed with plasmids pBAD-RFP, GIpF-dCas9 and pgRNA-RFP), 8739-RFP-CK-1 (8739 co-transformed with plasmids pBAD-RFP, GlpF-dCas9 and pgRNA-N20), 8739-RFP-CK-2(8739 co-transformed with pBAD-RFP, pTrc99A-M and pgRNA-RFP)

the expression of genes by binding to their promoter region [21], we tested whether the GlpF-dCas9 fusion still has the specific DNA binding activity guided by gRNA, the targeting sequence of dCas9 was located upstream of the $r f p$ gene.

To carry out the experiment, E. coli ATCC 8739 was co-transformed with the plasmids pBAD-RFP, pGlpFdCas9 and pgRNA-RFP, which was named 8739-RFP. A pgRNA-N20 with a random non-specific 20 bp sequence was constructed and used as the control, the strain was abbreviated to 8739-RFP-CK1. To exclude the effect of small RNA, another control strain 8739-RFP-CK2 was constructed, E. coli ATCC 8739 was co-transformed with the plasmids pBAD-RFP, pTrc99A-M and pgRNA-RFP. The RFP fluorescence intensity of the co-transformed cells was determined after IPTG induction for $24 \mathrm{~h}$. The fluorescence intensity of 8739-RFP-CK1 and 8739-RFPCK2 were similar, while compared with $8739-\mathrm{RFP}-C K 1$, the fluorescence intensity of 8739-RFP decreased significantly to $38.37 \%$ (Fig. 2), which indicated that the specific binding of GlpF-dCas9 to the target region guided by gRNA was functional, the binding of GlpF-dCas9 blocked the transcription of $r f p$ and reduced the expression of RFP, and the small gRNA alone did not affect the expression of RFP. Thus, the fusion of GlpF to dCas9 via 
the GGGS linker did not affect the binding activity of dCas9, and suggested a feasible binding of GlpF-dCas9 with its target plasmid.

\section{Localization of the crtW or crtZ expression cassettes using dCas9-Lag for improved canthaxanthin or zeaxanthin production}

The CrtW protein from Brevundimonas $s p$. SD212 has four transmembrane regions and CrtZ derived from Pantoea agglomerans has two transmembrane regions [22]. For the synthesis of canthaxanthin or zeaxanthin, these enzymes were expressed in E. coli. However, the efficiency of their targeting to the membrane compartment in heterologous hosts was unknown. We therefore attempted to use GlpF-dCas9 to localize the expression plasmid near the cell membrane and increase the functional expression of the enzymes as well as position them near their membrane-bound substrates.

The plasmids pSC101-CrtW and pSC101-CrtZ were individually introduced into the $\beta$-carotene production strain CAR025. To localize the expression cassettes of crt $W$ or $c r t Z$ to the membrane and not affect the transcription processes of $c r t Z$ and $c r t W$ genes, the binding locus of dCas9-Lag was selected at the backbone of plasmid pSC101, Subsequently, E. coli CAR025 was transformed with pgRNA-pSC101bb and pGlpF-dCas9, and pgRNA-N20 with a random non-specific 20 bp sequence was introduced as a control plasmid. The resulting transformed strains were designated as CAR025-CrtW, CAR025-CrtW-CK, CAR025-CrtZ and CAR025-CrtZ$C K$, respectively.

As shown in Fig. 3a, CAR025-CrtW produced $2.93 \mathrm{mg} / \mathrm{L}$ canthaxanthin with a specific production value of $0.54 \mathrm{mg} / \mathrm{g} \mathrm{DCW}$, representing an increase of 14.0 and $17.4 \%$ compared with the control strain
CAR025-CrtW-CK, respectively (Fig. 3a). CAR025CrtZ produced $4.51 \mathrm{mg} / \mathrm{L}$ zeaxanthin with the yield of $0.89 \mathrm{mg} / \mathrm{g}$ DCW, which was an increase of 18.8 and $29.0 \%$ compared with CAR025-CrtZ-CK (Fig. 3b). According to statistical analysis, the increase of zeaxanthin yield was significant $(\mathrm{P}<0.01)$ and the increase of zeaxanthin titer was obvious $(\mathrm{P}<0.05)$ which indicated that localizing the expression cassette of $\mathrm{CrtZ}$ to the membrane using dCas9-Lag improved zeaxanthin production. The increase of production was possibly caused by the increased functional expression of enzymes in the membrane and the reduced distance between enzymes and membrane-bound substrates.

Considering potential impacts on cell division and replication, cell growths and plasmid stability of CAR025-CrtZ and it's corresponding control strain were measured. As shown in Additional file 1: Fig. S1, similar growth status was observed in both control and experiment strain, suggesting locating of the expression plasmid did not affect cell growth. Plasmids in strains CAR025-CrtZ and CAR025-CrtZ-CK, were gradually lost during $96 \mathrm{~h}$ continuously cultivation, but they were relatively stable in the first $48 \mathrm{~h}$, less than $10 \%$ of plasmids were lost (Additional file 1: Fig. S2). During the culture process, with the consumption of nutrients and antibiotics, plasmids might have a tendency to be lost in strains gradually, so we collected the cells and detected the carotenoids within about $48 \mathrm{~h}$. It was found both strains had similar decrease curve of their plasmid, suggesting that locating plasmids to the membrane did not affect their stability.

In order to reveal the location of the CrtW and CrtZ, RFP was linked to the C-terminal of CrtW and CrtZ. The resulting plasmids pSC101-CrtW-RFP/pSC101CrtZ-RFP, pGlpF-dCas9 and pgRNA-pSC101bb were
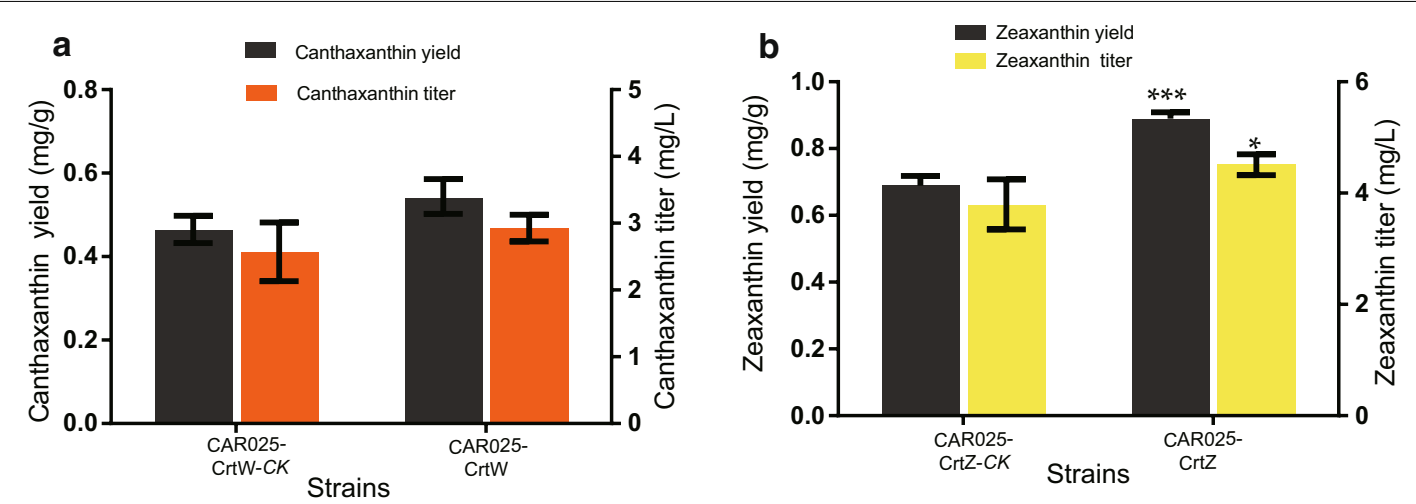

Fig. 3 Canthaxanthin and Zeaxanthin titer and yield of strain CAR025 with different pgRNA plasmids. a The Canthaxanthin titer and yield of CAR025. $\mathbf{b}$ The Zeaxanthin titer and yield of strain CAR025. Three repeats were performed for each strain, and the error bars represent standard deviation. The significance of the differences was calculated by Student's $t$-test; asterisks indicate significant differences compared with the control $\left({ }^{* *} \mathrm{P}<0.001 ;{ }^{* *} \mathrm{P}<0.01 ;{ }^{*} \mathrm{P}<0.05\right)$ 
expressed in CAR025. Compared with Cas9-Lag inactive strains, as shown in Additional file 1: Fig. S3, CrtZRFP and CrtW-RFP in Cas9-Lag strain were found more towards the edges areas of the cells, but the fluorescence microscopy images could not give definite location information due to the small size of $E$. coli cells.

\section{Localization of the combined cassette expressing both $c r t W$ and $c r t Z$ via dCas9-Lag for improved astaxanthin production}

The heterologous synthesis of astaxanthin from $\beta$-carotene is catalyzed by two enzymes, CrtW and CrtZ, which carry out four interchangeable reactions. The same vector pSC101 was used to express both $\operatorname{crt} W$ and $\operatorname{crtZ}$ via a constitutive promoter. To direct the plasmid carrying the synthetic pathway genes to the vicinity of the membrane compartment, pgRNA-pSC101bb and pGlpFdcas9 were introduced into CAR025(pSC101-CrtZW) to obtain the strain CAR025-CrtZW, similar to the previous single gene experiments. CAR025-CrtZW was able to produce $0.41 \mathrm{mg} / \mathrm{L}$ astaxanthin with a specific production value of $0.19 \mathrm{mg} / \mathrm{g}$ DCW, which was 24.2 and $26.7 \%$ higher than the corresponding values of the control strain CAR025-CrtWZ-CK (Fig. 4). The significant astaxanthin production improvement $(\mathrm{P}<0.01)$ of strain CAR025-CrtZW suggested the dCas9-Lag technique could increase astaxanthin production by localizing the combined cassette expressing the two genes $\operatorname{crt} W$ and crtZ.

\section{Protein mass spectrometry suggested increased CrtZ content in the membrane compartment}

To explore the mechanism of the increase of carotenoid production in the engineered strain in which the $\mathrm{crt}$ plasmids were targeted to the membrane using GlpF-dcas9, the membrane fraction was extracted and dissolve in $10 \%$ SDS, after which the genes were amplified by PCR with corresponding primers, and the proteins were analyzed by SDS-PAGE. However, the experimental groups did not show obvious gene concentration or an increase of protein expression in the membrane.

Subsequently, protein mass spectrometry was performed on the extracted membrane protein samples to determine if the content of membrane-bound $\mathrm{CrtW}$ and CrtZ protein was increased by dCas9-Lag. The detailed protein mass spectrometry results of $\mathrm{CrtW}$ and $\mathrm{CrtZ}$ are marked in red in tables S2-S5. The emPAI value is used to determine the relative protein abundance, while the Sum PEP Score, Score Sequest HT and PSMs values are used to determine the protein amount indirectly. While the emPAI values of the $\mathrm{CrtW}$ protein in the strains CAR025-CrtW and CAR025-CrtW-CK were both very low, the emPAI value of the CrtZ protein in the strain

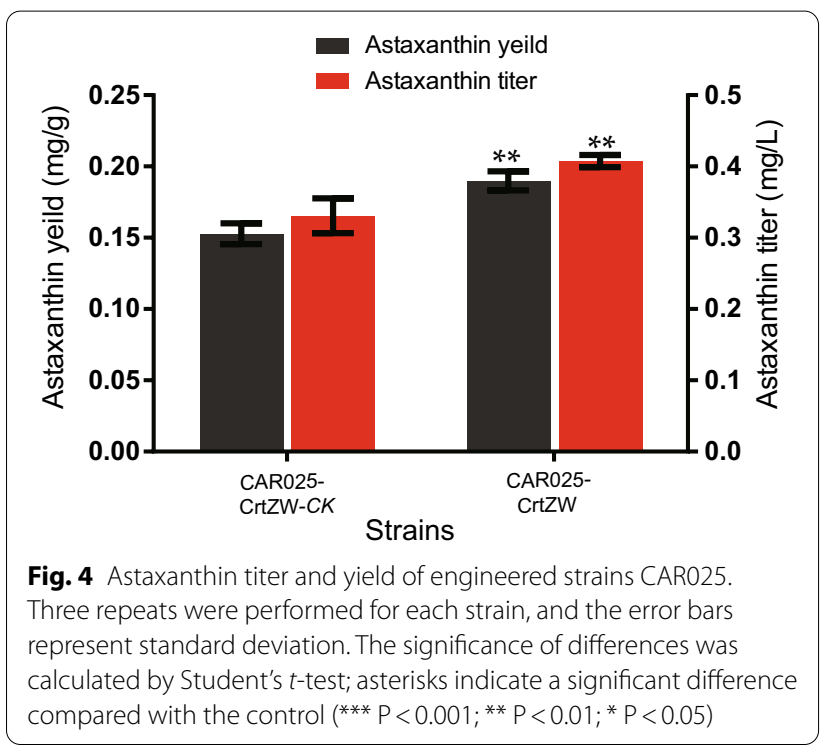

CAR025-CrtZ was obviously higher than in CAR025CrtZ-CK. Since mass spectral data was semi-quantitative, this result partially indicated that the expression of $\mathrm{CrtZ}$ protein in the membrane compartment might be improved by dCas9-Lag. And the increased amount of membrane-bound $\mathrm{CrtZ}$ might explain the increased zeaxanthin production by the CAR025-CrtZ strain.

According to the analysis of UniProt, CrtW from Brevundimonas sp. SD212 has four transmembrane helixes, and CrtZ from Pantoea agglomerans has only two transmembrane helixes. Based on this information, maybe the membrane binding capacity of $\mathrm{CrtW}$ is already strong in $E$. coli, while the membrane binding capacity of CrtZ could still be improved by the Cas9-Lag technique. This result is consistent with our previous research, that the yield of zeaxanthin was significantly increased by the fusion of CrtZ with membrane tag GlpF, but the fusion of $\mathrm{CrtW}$ with GlpF did not significantly improve the yield of canthaxanthin [19].

\section{Observation of changes in the chromosome after targeting a chromosomal cassette using dCas9-Lag}

Since the location of plasmids cannot be observed directly, we decided to pull the chromosomal DNA toward the cell membrane by GlpF-dCas9 for electron microscopy observation. The plasmid pgRNA-IdhALIdhAR was constructed with two gRNAs targeting sequences up- and down-stream of the $i d h \mathrm{~A}$ gene. The targeting sites were chosen at the chromosomal idh $\mathrm{A}$ sites of E. coli CAR005 and CAR015, which contain the crtEXYIB operon integration site. The plasmid GlpF-dCas9 was introduced into E. coli cells along with 
pgRNA-IdhAL-IdhAR.The strains with plasmid pgRNAN20 were employed as controls.

As illustrated in Fig. 5, cell morphology was observed in CAR005- pgRNA-IdhAL-IdhAR (Fig. 5a) and CAR015- pgRNA-IdhAL-IdhAR (Fig. 5b) compared with the control strains. Due to the interaction between GlpFdCas9 and the target gene $i d h A$, the distribution of the chromosome in the cells was closer to the membrane compartment at some points marked by red arrows. Due to experimental laminations, some of the microscopical images appeared to be vague, which somehow support the possibility that GlpF-Cas9 was functional and can mediate the localization of targeted DNA cassettes to the membrane. To determine if Cas9-Lag actually bind to IdhA gene and perturb the gene expression, we checked the transcriptional level of IdhA by RT-PCR. The relative RNA transcription levels of the IdhA fragments in CAR005 and CAR015 carrying dCas9-Lag were $14.10 \%$ and $8.58 \%$ of the parent strains, respectively, normalized by a $16 \mathrm{~S}$ internal control. This result confirmed the binding of Cas9-Lag to the IdhA gene loci.

\section{Discussion}

Eukaryotes contain cell organelles, such as mitochondria and chloroplasts, in which a relatively independent environment is separated from other cell compartments. These organelles have their own DNA expression cassettes to produce enzymes and proteins that perform specific tasks within the compartment. Such a naturally evolved strategy to bring DNA expression cassettes into the cellular factory where its gene product is needed was proved to significantly increase the efficiency of the related cellular functions. However, due to their simple cellular organization, prokaryotes do not have the luxury of such independent organelles. In this work, we constructed a dCas9-Lag technique, the gene products of the DNA cassette were located in their favorable environment, and close to their substrates. To localize plasmids or chromosomal DNA expression cassettes to the membrane compartment, dCas9 was fused with the $E$. coli membrane docking tag GlpF [20], and a gRNA was designed to target the backbone region of the DNA cassettes. This was done to pull the DNA expression cassettes into the proximity of the cell membrane, so that the transcription and translation process could be carried out near the membrane and the produced enzymes directly localized in membrane.

As a class of long-chain hydrophobic terpenoids, carotenoids and their derivatives are insoluble in water and accumulate in membrane-containing cellular compartments. The results indicate that the functional expression of membrane proteins was enhanced in the virtual compartment produced by GlpF-dCas9 and the carotenoid gene expression cassette, while the enzymes were also brought close to their substrates in the membrane. Thus, a carotenoid producing quasi-compartment was established. In this work, we determined that pGlpF-dcas9 induced the localization of the single enzyme expression cassette pSC101-CrtZ, which significantly increased the titer of the product of zeaxanthin by $29.0 \%$. Similarly, pGlpF-dcas9 induced the localization of the twin-enzyme expression cassette pSC101-CrtZW, which significantly increased the titer of astaxanthin by $26.7 \%$. These results suggested that the dCas9-Lag-induced quasi-compartment was functional, creating a relatively concentrated environment for the carotenoid genes to be translated into enzymes, where the enzymes are close to their favored environment and substrates. With such a manipulation, we might have created a virtual organelle in $E$. coli for the production of carotenoids.

To confirm that dCas9-Lag induced the generation of the quasi-compartment, we tried to obtain evidence that dCas9-Lag had attached the DNA cassette onto the membrane. However, no PCR products specific for the DNA cassette were obtained using the extracted cellular membrane as template, which indicated that the DNA cassette is either not contained within the membrane compartment, or that the hydrophilic DNA was lost or shredded during the membrane extraction process. This is perhaps unsurprising, since the negative charge of the DNA constructs makes them incompatible with the hydrophobic membrane environment, and the interaction between dCas9-Lag and the plasmid might be weak, and was probably destroyed during cell disruption and ultracentrifugation. Thus, even if the DNA cassettes were pulled close to the membrane by dCas9-Lag, they could not be detected in the cellular membrane fraction following the membrane extraction process. On the other hand, to observe the function of dCas9-Lag directly, we pulled the chromosomal DNA toward the cell membrane using GlpF-dCas9, and the migration of the chromosome toward the cell membrane mediated by GlpF-dCas9 was observed using electron microscopy, although some of the images were vague due to experimental limitations. Furthermore, the protein produced by the GlpF-dCas9bound DNA expression cassette was detected by mass spectrometry in the membrane compartment. These results all indicate that dCas9-Lag indeed successfully attached the DNA cassette to the membrane, which might have formed a cellular quasi-compartment beneficial for the function of the membrane-dependent carotenoid synthesis pathway.

Due to physical limitations and experimental limitations, the experiments performed were the best we could do to support our hypothesis that dCas9-Lag could pull DNA cassette to desired locations. And fortunately, these 
a

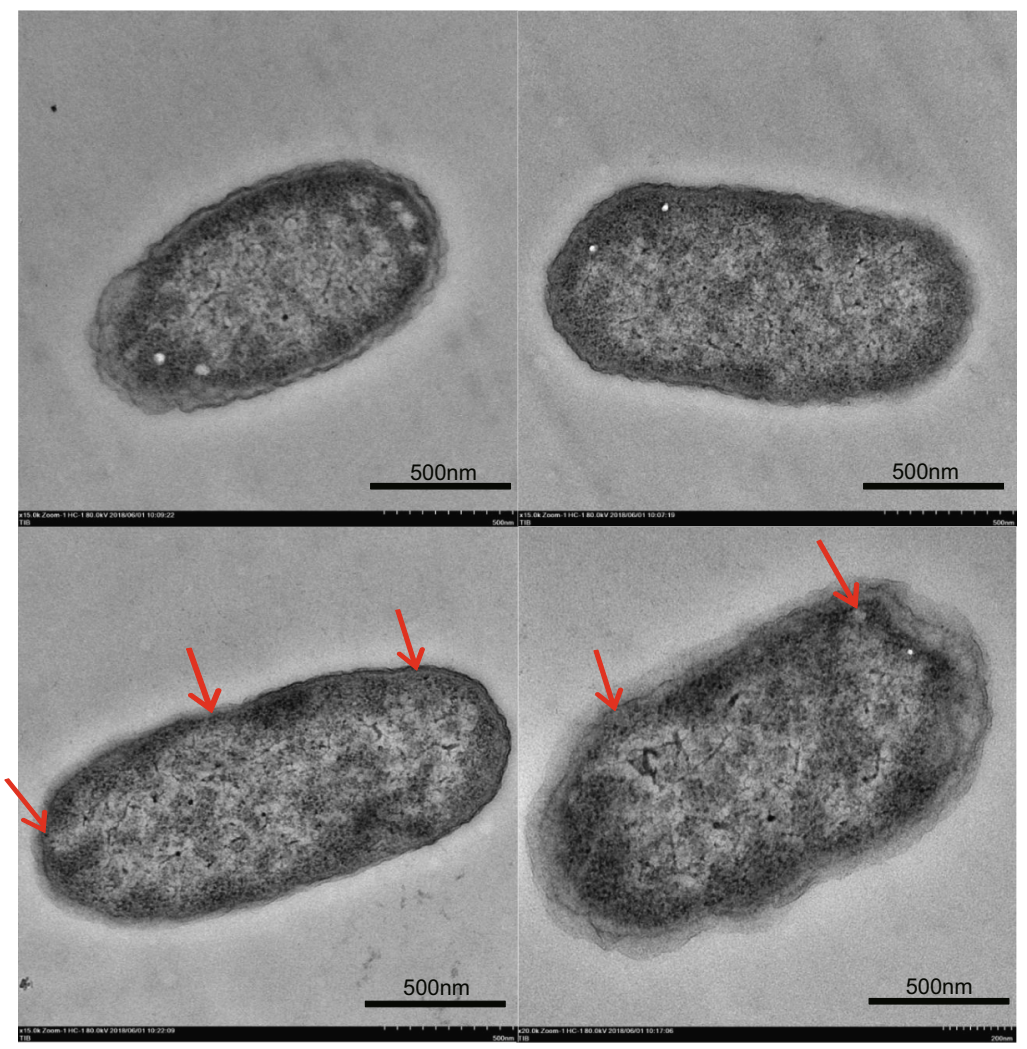

b

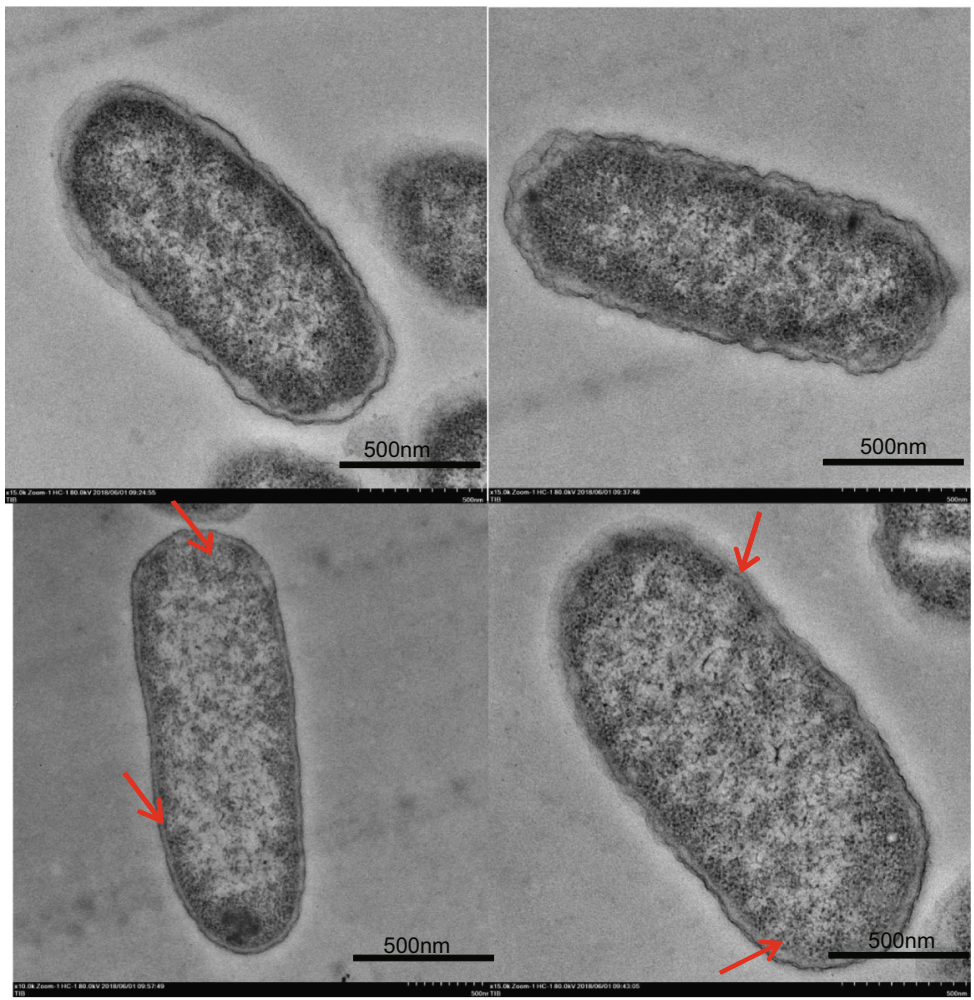

Fig. 5 Electron microscopic pictures of the genome binding to the membrane by GlpF-dcas 9 in derivatives of $\beta$-Carotene strains. a The change of genome distribution of CAR005. $\mathbf{b}$ The change of genome distribution of CAR015. Red arrows indicated the observed changes of membrane morphology, the distribution of the chromosome in CAR005 and CAR015 were closer to the membrane at some point as the Red arrows indicated 
experiments gave us positive but not $100 \%$ absolute evidences. However, we think the strategy presented in this article was very novel and interesting. We hope fellow researchers might be inspired and do more and deeper research with ideas similar to dCas9-Lag, which might also be used to improve their microbial factories.

\section{Conclusion}

A novel strategy was developed to fuse location tags to dCas9 (Cas9-Lag), and applied the technique for metabolic engineering. The zeaxanthin titer increased $29.0 \%$ when plasmid pSC101-CrtZ containing crtZ was localized to the membrane with Cas9-Lag, and the astaxanthin pathway product titer increased $26.7 \%$ with Cas9-Lag compared with control. This study established the novel molecular device Cas9-Lag and demonstrated that it might be able to manipulate the localization of DNA cassettes, which may have various future applications.

\section{Materials and methods}

\section{Strains, media and growth conditions}

The strains used in this study are listed in Table 1. All modified strains were derived from E. coli ATCC 8739. For seed cultures, single colonies were picked from plates and transferred into $15 \mathrm{~mm} \times 100 \mathrm{~mm}$ tubes containing LB with chloramphenicol $(30 \mathrm{mg} / \mathrm{L})$, apramycin $(50 \mathrm{mg} / \mathrm{L})$ and ampicillin $(100 \mathrm{mg} / \mathrm{L})$, and incubated at $37{ }^{\circ} \mathrm{C}$ and $250 \mathrm{rpm}$ overnight. The resulting seed cultures were subsequently used to inoculate $100 \mathrm{ml}$ flasks containing $10 \mathrm{ml}$ of fermentation medium with appropriate antibiotics to an initial optical density at $600 \mathrm{~nm}\left(\mathrm{OD}_{600}\right)$ of 0.05 , and grown at $30{ }^{\circ} \mathrm{C}$. IPTG was added to the culture after $3 \mathrm{~h}$ to a final concentration of $0.1 \mathrm{mM}$, and the cells were collected for measurement of products after $48 \mathrm{~h}$ of growth. The $\mathrm{OD}_{600}$ were measured constantly to determine the biomass concentration within $48 \mathrm{~h}$ using an SP-723 spectrophotometer (Spectrum SHANGHAI, China).

Table 1 Strains and plasmids used in this work

\begin{tabular}{|c|c|c|}
\hline Strains and plasmids & Relative characteristics & Resources \\
\hline \multicolumn{3}{|l|}{ Strains } \\
\hline ATCC8739 & Wild type & Lab collection \\
\hline CAR005 & ATCC 8739, M1-37::dxs, M1-46::idi M1-93::Crt, M1-46::SucAB, M1-46::sdh, M1-46::talB & Zhao et al. [11] \\
\hline CAR015 & CAR005, ispG-mRSL-4, ispH-mRSL-14 & Lab collection \\
\hline CAR025 & CAR015, replacing the promoter of $\mathrm{Cr}$ EYIB with Ptrc promoter & Lab collection \\
\hline CAR025-CrtW & CAR025 ( pSC101-CrtW,pGlpF-dCas9, pgRNA-pSC101bb) & This work \\
\hline CAR025-CrtW-CK & CAR025 (pSC101-CrtW,pGlpF-dCas9, pgRNA-N20) & This work \\
\hline CAR025-CrtZ & CAR025 (pSC101-CrtZ,pGlpF-dCas9, pgRNA-pSC101bb) & This work \\
\hline CAR025-CrtZ-CK & CAR025 (pSC101-CrtZ,pGlpF-dCas9, pgRNA-N20) & This work \\
\hline CAR025-CrtZW & CAR025 (pSC101-CrtZW,pGlpF-dCas9, pgRNA-pSC101bb) & This work \\
\hline CAR025-CrtZW-CK & CAR025 (pSC101-CrtZW,pGlpF-dCas9, pgRNA-N20) & This work \\
\hline 8739-RFP & ATCC 8739 (pBAD-RFP, pGlpF-dCas9, pgRNA-RFP) & This work \\
\hline 8739-RFP-CK1 & ATCC 8739 (pBAD-RFP, pGlpF-dCas9, pgRNA-N20) & This work \\
\hline 8739-RFP-CK2 & ATCC 8739 (pBAD-RFP, pTrc99A-M, pgRNA-N20) & This work \\
\hline \multicolumn{3}{|l|}{ Plasmids } \\
\hline pACYC184-M & cat; replace tet with lacl and Ptrc of pTrc99A-M & Zhao et al. [11] \\
\hline pCas9 & Cas9 expression plasmid & Zhao et al. (2016) \\
\hline pSC101 & low copy plasmid, ori and repA, M1-46 promoter, cat from pACYC184-M2-Pm46 & Lab collection \\
\hline pYL501 & crtW and crtZ in pSC101 & Lab collection \\
\hline pCrtW & crtW in pSC101 & This work \\
\hline $\mathrm{pCrtZ}$ & crtZ in pSC101 & This work \\
\hline pCrtZW & crtW and crtZ in pSC101 & This work \\
\hline pGlpF-dCas9 & GlpF fused with dCas9 in pTrc99A, pMB1 & This work \\
\hline pgRNA-RFP & Apr, p15A, gRNA-rfp 20 bp guide sequence(catgcgtttcaaagttcgta) & This work \\
\hline pgRNA-pSC101bb & Apr, p15A, gRNA-pYL501 bb 20 bp guide sequence(catgtaacacctaatagaac) & This work \\
\hline pgRNA-IdhAL-IdhAR & $\begin{array}{l}\text { Apr, p15A, gRNA-IdhAL-gRNA-IdhAR } 20 \text { bp guide sequence L(cagtaataacagcgcgagaa) } 20 \text { bp } \\
\text { guide sequence R(tgttgcgctaagcctgctga) }\end{array}$ & This work \\
\hline pBAD-RFP & Kna, pBAD-rfp, repA101 & Lab collection \\
\hline
\end{tabular}


Plasmid construction, transformation and plasmid stability assay

All plasmids were assembled using the Golden Gate DNA assembly method [23] and are listed in Table 1. The primers used in this study are summarized in Additional file 1: Table S1. All the primers were designed with linkers for the type II restriction enzyme BsaI for DNA assembly.

The backbone for the expression of the carotenoid synthesis pathway was amplified from the plasmid pSC101 and its derivative pYL501. Fragments containing $\operatorname{crtW}$ and $c r t Z$ were amplified from pYL501 [11]. The PCR products were subjected to DpnI digestion $(10 \mathrm{U}, 16 \mathrm{~h}$, $37{ }^{\circ} \mathrm{C}$ ) and gel purification before plasmid construction. The DNA fragment containing the dCas9 gene was obtained by mutating the Cas 9 gene (Addgene reference number 42876). GlpF was amplified from the genome of E. coli 8739 using the primer pair GlpF-F/GlpF-R and the two fragments were assembled into the pTrc99A$\mathrm{M}$ backbone. The linker between GlpF and dCas9 was GGGS [24]. Its nucleotide sequence GGCGGCGGCAGT , was divided into two parts and imbedded in the primers dCas9F and dCas9R separately.

For assembly of pgRNA plasmids, for example pgRNACrt, the N20 sequence (catgtaacacctaatagaac) was directly synthesized and embedded in the primers. The plasmid backbone contained the p15A replication origin and the apr gene. The plasmids pgRNA-RFP pgRNAIdhAL, pgRNA-IdhAR and pgRNA-IdhAL-IdhAR were constructed in analogy to pgRNA-Crt.

Plasmids were transformed into E. coil cells via electroporation, fifty microliters of competent cells were mixed with two or three plasmids (about $25 \mathrm{ng}$ each), which were electroporated at $2.5 \mathrm{kV}, 200 \Omega$ (Bio-Rad, Hercules, CA) and immediately incubated in $1 \mathrm{~mL}$ LB for $1 \mathrm{~h}$ at $37^{\circ} \mathrm{C}$, then plated on selective media.

For plasmid stability assay, overnight incubated fermentation medium was inoculated into $4 \mathrm{~mL}$ of fresh medium (LB containing $2 \%$ glycerol without any antibiotics), after $12 \mathrm{~h}$ growth in $30^{\circ} \mathrm{C}$, the culture transferred again, the experiment was conducted for a total of 8 transfers (approximately 6 generations per transfer). Different batches of cultures were serially diluted, plated on LB media, after overnight growth, 100 colonies were picked and streaked on selective media, colonies on the plate were counted as plasmid carrying.

\section{Analysis of RFP fluorescence intensity using a microplate reader}

The RFP-expressing colonies were picked and transferred into $3 \mathrm{~mL}$ of $\mathrm{LB}$ with $50 \mathrm{mg} / \mathrm{L}$ of kanamycin, $50 \mathrm{mg} / \mathrm{L}$ apramycin and $100 \mathrm{mg} / \mathrm{L}$ ampicillin, and grown at $37{ }^{\circ} \mathrm{C}$ and $250 \mathrm{rpm}$ overnight. The cultures were then transferred into $15 \mathrm{~mm} \times 100 \mathrm{~mm}$ tubes containing $3 \mathrm{~mL}$ of LB with the same antibiotics and grown at $37{ }^{\circ} \mathrm{C}$ and $250 \mathrm{rpm}$ for $20 \mathrm{~h}$. Subsequently, samples of each culture were diluted with LB four times and $200 \mu \mathrm{L}$ of them transferred into individual wells of a 96-well plate. The blank control was $200 \mu \mathrm{L}$ of pure LB. Fluorescence was measured at an excitation wavelength of $585 \mathrm{~nm}$ and an emission wavelength of $620 \mathrm{~nm}$ using an Infinite M200 Pro ELISA spectrometer (Tecan, Switzerland).

\section{Observation of cell morphology via transmission electron microscopy}

After $48 \mathrm{~h}$ of cultivation, cells were collected, washed three times with phosphate buffered saline (PBS, pH 7.4), and fixed with $1 \%$ glutaraldehyde at $4{ }^{\circ} \mathrm{C}$ overnight. The fixed cells were resuspended in $1 \%$ osmic acid and incubated for $5 \mathrm{~min}$ at room temperature, and then resuspended again in fresh $1 \%$ osmic acid and incubated for $45 \mathrm{~min}$ at room temperature. Then the cells were dehydrated by immersion in graded ethanol solutions $(50 \%$, $70 \%, 80 \%, 90 \%, 95 \%$, and $100 \%$ ) for 15 min each. For embedding, cells were infiltrated by incubating for $2 \mathrm{~h}$ in 3:1, 1:1, and 1:3 embedding medium. Then, the cells were resuspended in pure embedding medium and incubated at room temperature overnight. The next day, cells were resuspended in fresh embedding medium and cured for 24 to $48 \mathrm{~h}$ at $80{ }^{\circ} \mathrm{C}$, cut into 60 to $80 \mathrm{~nm}$ sections, stained with uranyl acetate and lead citrate, and examined under a Hitachi HT7700 electron microscope (Hitachi, Japan) operating at $180 \mathrm{kV}[25,26]$.

Measurement of carotenoids produced by the cell factories To quantify the production of astaxanthin, zeaxanthin and canthaxanthin, samples comprising $1 \mathrm{~mL}$ of culture broth were centrifuged at $16,200 \times \mathrm{g}$ for $3 \mathrm{~min}$, and the cell pellet was washed with sterile water. Subsequently, $750 \mu \mathrm{L}$ of extraction solution (acetonitrile/methanol/ dichloromethane, 21:21:8, v/v/v) was added to the pellet and ultrasonicated in an ice bath for $30 \mathrm{~min}$, after which the resulting lysate was centrifuged at $16,200 \times \mathrm{g}$ for $3 \mathrm{~min}$. The resulting supernatant was collected and another $750 \mu \mathrm{L}$ of extraction solution was added to repeat the extraction [27]. The supernatants of both extraction steps were combined, centrifuged at $16,200 \times \mathrm{g}$ for $3 \mathrm{~min}$, and filtered through a $0.22 \mu \mathrm{m}$ organic nylon filter before analysis by high-performance liquid chromatography (HPLC) using an Agilent Series 1200 system with a variable wavelength detector set at $476 \mathrm{~nm}$ and a Symmetry C18 column ( $250 \mathrm{~mm} \times 4.6 \mathrm{~mm}, 5 \mu \mathrm{m}$, Waters, Ireland). The carotenoid detection method was the same as reported before $[11,28]$. The results represent the means of three independent experiments. The reference standards of the indicated compounds were purchased from 
Sigma (Sigma-Aldrich, USA). Dry cell weight (DCW) was calculated according to the empirical formula: 1 $\mathrm{OD}_{00}=0.323 \mathrm{~g} \mathrm{DCW} / \mathrm{L}$.

\section{Extraction of cell membranes}

Membrane extraction was performed according to a previously reported protocol [29]. Samples comprising $50 \mathrm{~mL}$ of fermentation broth were collected after $48 \mathrm{~h}$ of cultivation at $30{ }^{\circ} \mathrm{C}$ and resuspended in buffer $(50 \mathrm{mM}$ Tris- $\mathrm{HCl} \mathrm{pH}$ 7.5, $150 \mathrm{mM} \mathrm{NaCl}$ ). Cells were lysed using a French Press (JN-3000plus, China) at 1200 bar, and debris was removed by centrifugation at $10,000 \mathrm{~g}$ for $15 \mathrm{~min}$. The supernatant was collected and centrifuged at $210,000 \mathrm{~g}$ for $1 \mathrm{~h}$ using a Beckman Optima L-100XP ultracentrifuge equipped with a Beckman-Coulter $41 \mathrm{Ti}$ rotor (Beckman-Coulter, Germany). The membrane fraction was collected at the bottom of the centrifuge tube as a pellet, and then dissolved using denaturation buffer (8 M urea, $1 \%$ DTT) and stored at $-80{ }^{\circ} \mathrm{C}$ for mass spectrometry or further analysis.

\section{Statistical analysis and analytical techniques}

The significance of differences between mean values of control and test samples was assessed using Student's $t$-test in the open-source software suite "R" (http://cran.rproject.org/). Differences with $p<0.05$ were regarded as obvious, $p<0.01$ as significant, and $p<0.001$ as very significant. The SDS-PAGE was run using the commercially purchased SurePage ${ }^{\mathrm{TM}}$ Gels (GenScript, Nanjing). The protein mass spectrometry was performed using an OrbiTrap Fusion LUMOS Tribrid Mass Spectrometer (LC-MS) (Thermo Fisher, USA) according to published methods $[3,30]$.

\section{Supplementary Information}

The online version contains supplementary material available at https://doi. org/10.1186/s12934-020-01496-w.

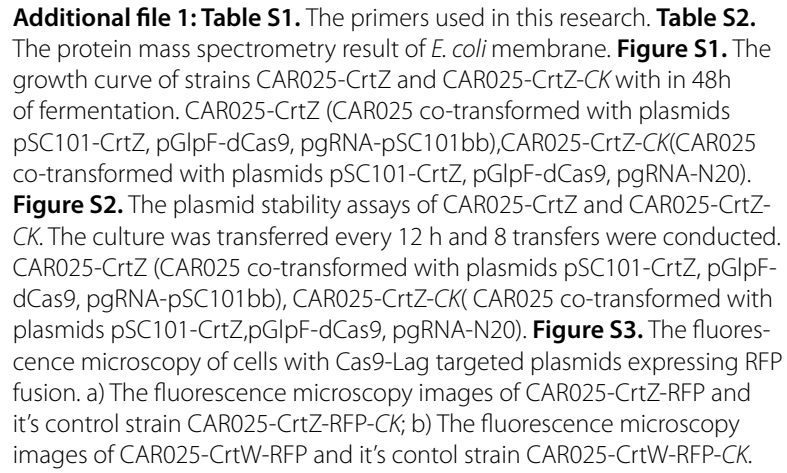

Additional file 1: Table S1. The primers used in this research. Table S2. The protein mass spectrometry result of E. coli membrane. Figure S1. The growth curve of strains CAR025-CrtZ and CAR025-CrtZ-CK with in 48h of fermentation. CAR025-CrtZ (CAR025 co-transformed with plasmids pSC101-CrtZ, pGlpF-dCas9, pgRNA-pSC101bb),CAR025-CrtZ-CK(CAR025 Co-transformed with plasmids pSC101-CrtZ, pGlpF-dCas9, pgRNA-N20). Figure S2. The plasmid stability assays of CAR025-CrtZ and CAR025-CrtZCK. The culture was transferred every $12 \mathrm{~h}$ and 8 transfers were conducted. CAR025-CrtZ (CAR025 co-transformed with plasmids pSC101-CrtZ, pGlpFdCas9, pgRNA-pSC101bb), CAR025-CrtZ-CK( CAR025 co-transformed with plasmids pSC101-CrtZ,pGlpF-dCas9, pgRNA-N20). Figure S3. The fluorescence microscopy of cells with Cas9-Lag targeted plasmids expressing RFP fusion. a) The fluorescence microscopy images of CAR025-CrtZ-RFP and it's control strain CAR025-CrtZ-RFP-CK; b) The fluorescence microscopy images of CAR025-CrtW-RFP and it's contol strain CAR025-CrtW-RFP-CK.

\section{Acknowledgements}

Not applicable.

\section{Authors' contributions}

QX and SL planned and performed experiments, analyzed and interpreted the data. CB and $L Z$ supervised the study and interpreted the results. All authors read and approved the final manuscript.

\section{Funding}

This research was financially supported by The National Key Research and Development Program of China (2018YFA0903700), Tianjin Synthetic Biotechnology Innovation Capacity Improvement Project (TSBICIP-KJGG-001) and National Natural Science Foundation of China (31770105).

\section{Availability of data and materials}

All data generated or analyzed during this study are included in this published article and its Additional file 1.

\section{Ethics approval and consent to participate}

Not applicable.

\section{Consent for publication}

I hereby give the Journal of Microbial Cell Factories the right and permission to publish this article.

\section{Competing interests}

The authors declare that they have no competing interests.

\section{Author details}

${ }^{1}$ College of Biotechnology, Tianjin University of Science and Technology, Tianjin 300457, P. R. China. ${ }^{2}$ State Key Laboratory of Pathogen and Biosecurity, Beijing Institute of Biotechnology, Beijing 100071, China. ${ }^{3}$ Tianjin Institute of Industrial Biotechnology, Chinese Academy of Sciences, Tianjin 300308, P. R. China. ${ }^{4}$ Key Laboratory of Systems Microbial Biotechnology, Chinese Academy of Sciences, Tianjin 300308, China.

Received: 26 September 2020 Accepted: 5 December 2020

Published online: 14 December 2020

\section{References}

1. Cong L, Ran FA, Cox D, Lin S, Barretto R, Habib N, Hsu PD, Wu X, Jiang W, Marraffini LA, Zhang F. Multiplex genome engineering using CRISPR/Cas systems. Science. 2013;339:819-23.

2. Cho SW, Kim S, Kim JM, Kim JS. Targeted genome engineering in human cells with the Cas9 RNA-guided endonuclease. Nat Biotechnol. 2013;31:230-2.

3. Espadas G, Borras E, Chiva C, Sabido E. Evaluation of different peptide fragmentation types and mass analyzers in data-dependent methods using an Orbitrap Fusion Lumos Tribrid mass spectrometer. Proteomics. 2017:17:1600416.

4. Chen B, Gilbert LA, Cimini BA, Schnitzbauer J, Zhang W, Li GW, Park J, Blackburn EH, Weissman JS, Qi LS, Huang B. Dynamic imaging of genomic loci in living human cells by an optimized CRISPR/Cas system. Cell. 2013:155:1479-91.

5. Xia AL, He QF, Wang JC, Zhu J, Sha YQ, Sun B, Lu XJ: Applications and advances of CRISPR-Cas9 in cancer immunotherapy. J Med Genet. 2018.

6. Gao Y, Xiong X, Wong S, Charles EJ, Lim WA, Qi LS. Complex transcriptional modulation with orthogonal and inducible dCas9 regulators. Nat Methods. 2016;13:1043-9.

7. Gao X, Tsang JC, Gaba F, Wu D, Lu L, Liu P. Comparison of TALE designer transcription factors and the CRISPR/dCas9 in regulation of gene expression by targeting enhancers. Nucleic Acids Res. 2014;42:e155.

8. Ma Y, Zhang J, Yin W, Zhang Z, Song Y, Chang X. Targeted AID-mediated mutagenesis (TAM) enables efficient genomic diversification in mammalian cells. Nat Methods. 2016;13:1029-35.

9. Schmidtdannert C. Engineering novel carotenoids in microorganisms. Curr Opin Biotechnol. 2000;11:255.

10. Das A, Yoon SH, Lee SH, Kim JY, Oh DK, Kim SW. An update on microbial carotenoid production: application of recent metabolic engineering tools. Appl Microbiol Biotechnol. 2007;77:505-12. 
11. Zhao J, Li Q, Sun T, Zhu X, Xu H, Tang J, Zhang X, Ma Y. Engineering central metabolic modules of Escherichia coli for improving beta-carotene production. Metab Eng. 2013;17:42-50.

12. Jin YS, Stephanopoulos G. Multi-dimensional gene target search for improving lycopene biosynthesis in Escherichia coli. Metab Eng. 2007:9:337-47.

13. Choi SK, Matsuda S, Hoshino T, Peng X, Misawa N. Characterization of bacterial beta-carotene 3,3'-hydroxylases, CrtZ, and P450 in astaxanthin biosynthetic pathway and adonirubin production by gene combination in Escherichia coli. Appl Microbiol Biotechnol. 2006;72:1238-46.

14. Misawa N, Satomi Y, Kondo K, Yokoyama A, Kajiwara S, Saito T, Ohtani T, Miki W. Structure and functional analysis of a marine bacterial carotenoid biosynthesis gene cluster and astaxanthin biosynthetic pathway proposed at the gene level. J Bacteriol. 1995;177:6575-84

15. Ahrazem O, Rubio-Moraga A, Berman J, Capell T, Christou P, Zhu C, Gomez-Gomez L. The carotenoid cleavage dioxygenase CCD2 catalysing the synthesis of crocetin in spring crocuses and saffron is a plastidial enzyme. New Phytol. 2016;209:650-63.

16. Wu T, Ye L, Zhao D, Li S, Li Q, Zhang B, Bi C, Zhang X. Membrane engineering - a novel strategy to enhance the production and accumulation of beta-carotene in Escherichia coli. Metab Eng. 2017;43:85-91.

17. Farhi M, Marhevka E, Masci T, Marcos E, Eyal Y, Ovadis M, Abeliovich H, Vainstein A. Harnessing yeast subcellular compartments for the production of plant terpenoids. Metab Eng. 2011;13:474-81.

18. Lv X, Wang F, Zhou P, Ye L, Xie W, Xu H, Yu H. Dual regulation of cytoplasmic and mitochondrial acetyl-CoA utilization for improved isoprene production in Saccharomyces cerevisiae. Nat Commun. 2016;7:12851.

19. Ye LJ, Zhu XN, Wu T, Wang W, Zhao DD, Bi CH, Zhang XL. Optimizing the localization of astaxanthin enzymes for improved productivity. Biotechnol Biofuels. 2018;11:278.

20. Neophytou I, Harvey R, Lawrence J, Marsh P, Panaretou B, Barlow D. Eukaryotic integral membrane protein expression utilizing the Escherichia coli glycerol-conducting channel protein (GlpF). Appl Microbiol Biotechnol. 2007;77:375-81.

21. Cress BF, Jones JA, Kim DC, Leitz QD, Englaender JA, Collins SM, Linhardt RJ, Koffas MAG. Rapid generation of CRISPR/dCas9-regulated, orthogonally repressible hybrid T7-lac promoters for modular, tuneable control of metabolic pathway fluxes in Escherichia coli. Nucleic Acids Res. 2016:44:4472-85.

22. Fraser PD, Miura Y, Misawa N. In vitro characterization of astaxanthin biosynthetic enzymes. J Biol Chem. 1997;272:6128-35.

23. Engler C, Gruetzner R, Kandzia R, Marillonnet S. Golden gate shuffling: a one-pot DNA shuffling method based on type lls restriction enzymes. PLOS ONE. 2009;4:e5553.

24. Chen $X Y$, Zaro JL, Shen WC. Fusion protein linkers: Property, design and functionality. Adv Drug Deliv Rev. 2013;65:1357-69.

25. Herskovits AA, Shimoni E, Minsky A, Bibi E. Accumulation of endoplasmic membranes and novel membrane-bound ribosome-signal recognition particle receptor complexes in Escherichia coli. J Cell Biol. 2002;159:403-10.

26. Wilfling F, Wang HJ, Haas JT, Krahmer N, Gould TJ, Uchida A, Cheng JX, Graham M, Christiano R, Frohlich F, et al. Triacylglycerol synthesis enzymes mediate lipid droplet growth by relocalizing from the ER to lipid droplets. Dev Cell. 2013;24:384-99.

27. Lemuth K, Steuer K, Albermann C. Engineering of a plasmid-free Escherichia coli strain for improved in vivo biosynthesis of astaxanthin. Microb Cell Fact. 2011;10:29.

28. Tao L, Wilczek J, Odom JM, Cheng Q. Engineering a beta-carotene ketolase for astaxanthin production. Metab Eng. 2006;8:523-31.

29. Lu P, Ma D, Yan C, Gong X, Du M, Shi Y. Structure and mechanism of a eukaryotic transmembrane ascorbate-dependent oxidoreductase. Proc Natl Acad Sci U S A. 2014;111:1813-8.

30. Li S, Zhou Y, Xiao K, Li J, Tian Z. Selective fragmentation of the N-glycan moiety and protein backbone of ribonuclease B on an Orbitrap Fusion Lumos Tribrid mass spectrometer. Rapid Commun Mass Spectrom. 2018;32:2031-9.

\section{Publisher's Note}

Springer Nature remains neutral with regard to jurisdictional claims in published maps and institutional affiliations.
Ready to submit your research? Choose BMC and benefit from:

- fast, convenient online submission

- thorough peer review by experienced researchers in your field

- rapid publication on acceptance

- support for research data, including large and complex data types

- gold Open Access which fosters wider collaboration and increased citations

- maximum visibility for your research: over $100 \mathrm{M}$ website views per year

At BMC, research is always in progress.

Learn more biomedcentral.com/submissions 